\title{
Accuracy, completeness and accessibility of online information on fibromyalgia
}

\author{
Deepika Basavakumar $^{1} \cdot$ Mirika Flegg $^{2} \cdot$ Jessica Eccles $^{1}$ (I) $\cdot$ Pietro Ghezzi $^{1}$ (I)
}

Received: 17 January 2019 / Accepted: 25 February 2019 / Published online: 6 March 2019

(c) The Author(s) 2019

\begin{abstract}
Fibromyalgia is a multi-factorial illness primarily characterised by widespread chronic pain and fatigue, with several symptoms and associated conditions. Due to a lack of clinical awareness and an absence of objective diagnostic measures, fibromyalgia patients often engage with online health information. The aim is to investigate the completeness and trustworthiness of the information available online on fibromyalgia. Google.co.uk was searched for 'fibromyalgia', the first 200 webpages were imported and 148 were analysed for standard health information quality criteria (JAMA score, HONcode) as well as completeness of information in terms of symptoms, causes and treatments mentioned. The most frequent typology of webpages was from health professionals (38\%), with commercial websites being less frequent (7\%). Overall, the quality, completeness and accessibility of online health information was poor. Completeness of coverage for symptoms, causes and associated conditions was especially lacking, with pages from not-for-profit organisations discussing the highest number of symptoms (median $8, \min 0$, max 11 , interquartile range, IQR $4.5 ; n=14$ ) compared to the rest of the websites in the search engine results (median 4, $\min 0, \max 11, \mathrm{IQR} 4 ; n=134$ ). Mean readability was grade 9 (median 9, $\min 1, \max 18$, IQR 3), with only $8 \%$ websites meeting the recommended readability of grade 6 . The Internet provides incomplete information on fibromyalgia, which does not fulfil the most queried aspect(s) by patients, symptoms, and may be difficult to understand by lay persons. Not-for-profit organisations provide the most complete information compared to other types of websites.
\end{abstract}

Keywords Fibromyalgia $\cdot$ Pain $\cdot$ Information quality $\cdot$ Internet

\section{Introduction}

Fibromyalgia is a multi-factorial illness primarily characterised by widespread chronic pain and fatigue [1-6]. It is also associated with cognitive impairments [7] and affective symptoms such as changes in mood, depression and anxiety disorders [2-5, 7]. Associated systemic problems include irritable bowel syndrome/change of bowel habit and bladder symptoms $[2,4,7]$. Headaches, sleep and skin disturbances are also reported, as are coordination problems, muscle weakness, hair loss, fever, dry eyes and mouth and sensory sensitivity [2]. It is estimated that between 1 in 20 [8] and 1 in 25 [9] individuals are affected. Many more may suffer from fibromyalgia in the absence of a formal diagnosis, due to its complex and heterogeneous expression [5], a lack of clinical awareness and an absence of objective diagnostic measures [10]. Therefore, individuals with fibromyalgia can be easily dismissed, stigmatised and left alone to selfmanage their illness [10-12]. Fibromyalgia patients report a preference for engaging with online health information 
[13]. Associated stigma and engagement preferences may account for recent reports suggesting 'fibromyalgia' as the most common medical search term within the UK [14], with 'fibromyalgia symptoms' of primary interest according to Google trends on 14/8/2018.

The availability of online health information may help to improve patient engagement with professionals and treatment options $[1,15]$. This can be empowering to patients, but many argue that the quality of online information is often poor and presented in ways that can increase the likelihood that content may be misunderstood $[1,16,17]$. To the best of our knowledge, only one 2011 study has considered online health information specifically for fibromyalgia patients, analysing 25 webpages for quality and readability [1]. Content analysis was restricted to reporting on types of information included rather than considering the completeness of content. Information was found to be inconsistent, incomplete and open to misinterpretation, with the author recommending that health professionals ought to assist fibromyalgia patients in accessing quality information [1]. This study was designed to build on and address key gaps in knowledge of online health information quality on fibromyalgia, with two aims. The first aim of the study was to understand the completeness of the information in terms of symptoms, causes, associated conditions and treatment coverage. The second aim was to assess the trustworthiness in terms of standard health information quality instruments such as the Journal of American Medline Association (JAMA) score [16] and the presence of the Health-on-the-Net (HON) certification [18], as well as their readability.

\section{Methods}

A search of google.co.uk was carried out in October 2018 from Brighton, UK, using the search term "fibromyalgia". We used a neutral term to have a broader sample of the webpages on this topic. To limit personalisation of search results due to the potential influence of previous browsing history, we cleared cookies and browser history immediately before the search. We used Google, as it is the primary search engine of $80 \%$ of individuals in the UK [19]. The first 200 results were downloaded and visited, as this sample size chosen as in previous studies was found adequate to perform statistical analysis [20,21]. Of these, 52 were excluded based on exclusion criteria. These included the need for registration or payment to access the website, dead pages or pages with irrelevant information (for instance, this page that has no reference to fibromyalgia was returned: https:// www.mclaren.org/centralmichigan/health-information/6887. aspx). We also excluded fora and dynamic pages or indexes generated ad hoc (e.g.: https://www.pinterest.co.uk/explore/ fibromyalgia/). The remaining 148 webpages were analysed as described in the flowchart in Fig. 1.

We classified webpages according to the typologies of the website with webpages not fitting any of those classified as "others". The typologies used were the following: commercial, C (websites selling products or services, e.g. amazon.co.uk, igennus.com); government, G (e.g. nhs.uk, womenshealth.gov); health portals, HP (websites providing a wide range of health information only, e.g. webmd. com, thehealthsite.com); news, N (e.g. theguardian.com, bbc.co.uk); Not-for-profit, NP (e.g. ukfibromyalgia.com, arthritiscare.org.uk); professional, P (organisations or individuals with professional medical qualification, e.g. mayoclinic.org, circlehealth.co.uk); scientific journal, S (academic publications, e.g. bmj.com, jneurosci.org).

Webpages were then analysed as follows. Firstly, we explored the completeness of the fibromyalgia information in terms of symptoms, causes, associated conditions and treatment coverage. Secondly, we assessed trustworthiness in terms of standard health information quality instruments such as the Journal of American Medline Association (JAMA) score [16] and the presence of HONCode certification [18], as well as their readability. For content analysis, mention of the following was recorded: symptoms, causes, associated conditions and therapies. These lists were compiled based on the content analysis, and only headings mentioned at least five times were considered.

To assess standard information quality measures, webpages were then given a JAMA (1-4) score based on the presence of four criteria (indication of date, references/ sources, authorship and ownership) [16]. The presence of the HONcode quality stamp [18] or the Information Standard stamp accredited by the UK National Health Service was also recorded. If a criterion was not visible on the initial web page, the three-click rule was used, where if a specific feature could not be found within three clicks, the web page was given a score of 0 for that criterion. Web page readability was assessed using a standardised online tool (https:// www.webfx.com/tools/read-able/) as described previously [20] and expressed as reading grade (the higher the grade, the more difficult).

Statistical analysis was performed with GraphPad Prism 7.04 for Windows using the tests described in the text. The number of symptoms mentioned in a specific typology of websites was compared with the rest of the search, rather than the whole search, to avoid comparing overlapping data. To compare two groups, non-parametric tests were used, a two-tailed Mann-Whitney test for comparing two groups and a two-tailed Kruskal-Wallis test in case of multiple comparisons. Frequency of typologies of websites in the first ten results in the Google search were compared with that in the rest of the search, using a twotailed Fisher's exact test. The level of significance was set 


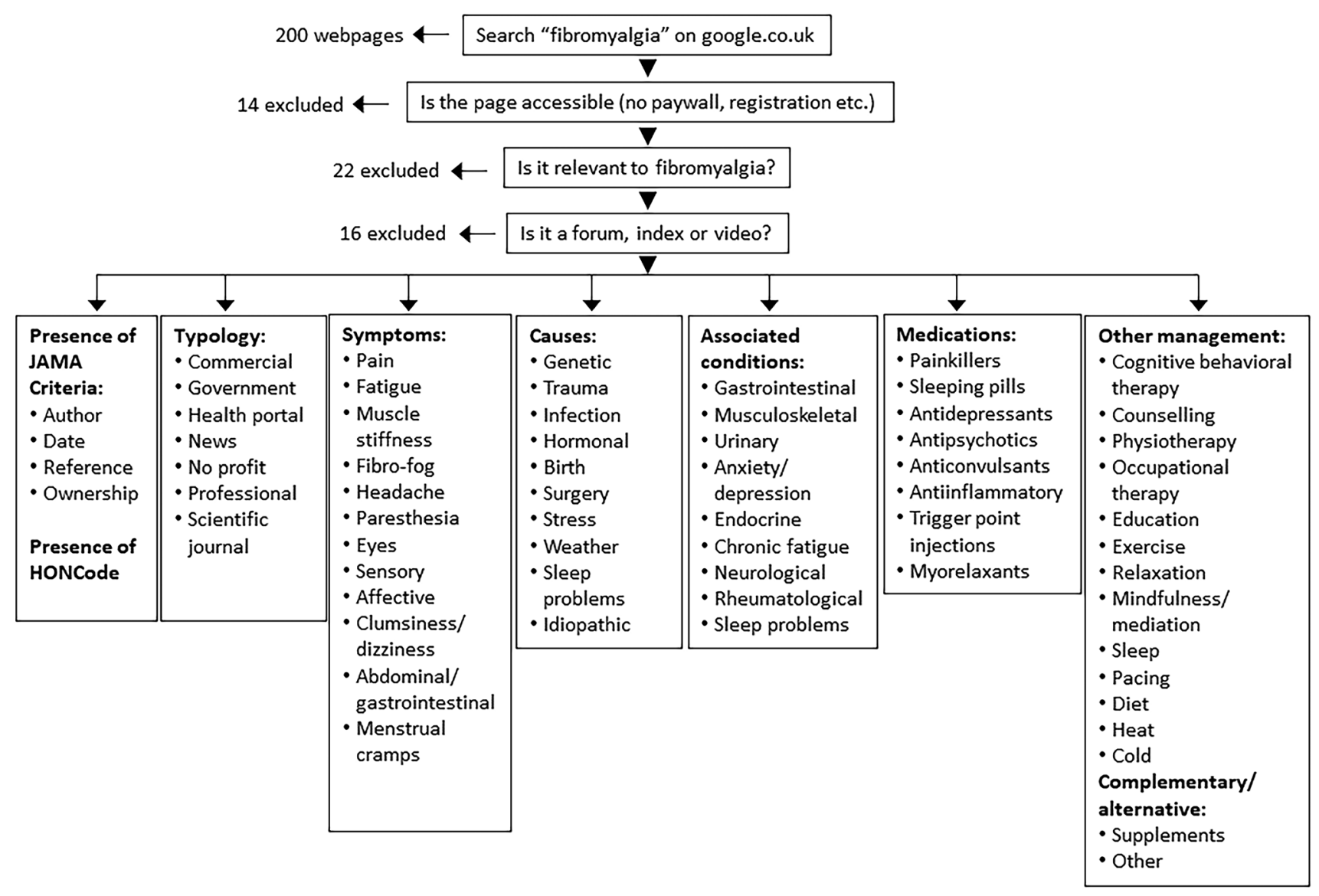

Fig. 1 Flowchart describing the analysis of the webpages

at $P<0.05$. Ethical approval was not sought because this research did not involve human participants.

\section{Results}

\section{Composition of the search engine result page (SERP)}

Of the 148 webpages analysed, most (56\%) were professional websites (hospitals, universities, professionals with a degree in a medical speciality), with all other typologies equally represented (Table 1). Interestingly, in the top ten results from the Google SERP, professional websites were not present, but websites from news outlets and not-forprofit organisations were more represented in comparison to the SERP as a whole. However, this should be only considered a trend, as none of these differences were statistically significant when the frequency in the top ten results was compared with that in the rest of the SERP using a two-tailed Fisher's exact test.
Table 1 Typology of websites in the search engine result page

\begin{tabular}{lll}
\hline Typology & $\begin{array}{l}\text { No. in whole search } \\
(\%)\end{array}$ & No. in top 10 (\%) \\
\hline Professional & $55(37 \%)$ & $0(0 \%)$ \\
News & $16(11 \%)$ & $3(30 \%)$ \\
Government & $14(9 \%)$ & $1(10 \%)$ \\
Not-for-profit & $14(9 \%)$ & $3(30 \%)$ \\
Scientific journal & $14(9 \%)$ & $0(0 \%)$ \\
Health portal & $12(8 \%)$ & $1(10 \%)$ \\
Other & $12(8 \%)$ & $1(10 \%)$ \\
Commercial & $11(7 \%)$ & $1(10 \%)$ \\
Total & $n=148$ & $n=10$
\end{tabular}

Numbers indicate the number of websites; percentage of total is indicated in parentheses

\section{Completeness of information: symptoms, causes and associated conditions}

Table 2 reports the most mentioned symptoms, causes and associated conditions. Across webpages, a total of 12 
Table 2 Content analysis: symptoms, causes and associated conditions mentioned

\begin{tabular}{lllll}
\hline Symptoms & No. $(\%)$ & Causes & No. $(\%)$ & Associated conditions \\
\hline Pain & 132 & Stress & 49 & Sleep disorders \\
Fatigue & 108 & Trauma & 47 & Rheumatology \\
Fibro fog & 77 & Infection & 36 & Anxiety/depression \\
Muscle stiffness & 70 & Genetics & 34 & Urinary \\
Headache & 65 & Idiopathic & 26 & Chronic fatigue syndrome \\
Affective & 61 & Hormonal & 20 & Musculoskeletal \\
Abdominal pain/gastrointestinal & 61 & Sleep disturbance & 19 & Gastrointestinal \\
Paraesthesia & 34 & Surgery & 15 & Endocrinological \\
Sensitivity to sensory stimulus & 27 & Giving birth & 12 & Neurological \\
Painful menstrual cramps & 23 & Weather & 12 & \\
Clumsiness/dizziness & 14 & & & \\
Eyes & 14 & & & \\
\hline
\end{tabular}

Data indicate the number of websites mentioning the specific item $(n=148)$. Only items that were described at least five times were included in the analysis. Percentages (in parentheses) are calculated on the total number of websites (148)

symptoms were reported, with pain and fatigue the most frequently mentioned. We then analysed each webpage to find how many of these symptoms, causes and associated conditions were mentioned, to assess its completeness. The frequency distribution of webpages according to their completeness is reported in Fig. 2. It can be seen that symptoms (Fig. 2a) have the most complete coverage (median 5, min 0 , max 11, IQR 5), although 31 webpages (21\%) mentioned only zero to one symptoms. Coverage of causes (Fig. 2b) or associated conditions (Fig. 2c) was lower, with very few websites mentioning more than one (causes, median 1 , min 0 , max 10, IQR 4; associated conditions, median 1, min 0 , $\max 7$, IQR 2).

Because symptoms are the most frequently searched content, we analysed their coverage across different typologies. Websites from not-for-profit organisations had the highest number of symptoms described (median $8, \min 0, \max 11$, IQR $4.5 ; n=14$ ). These were followed by news (median 5.5, $\min 0$, max, 11, IQR $3.5 ; n=16$ ), health portals (median 5.5, min 1, $\max 9$, IQR 6; $n=12$ ); commercial (median 5, min 0 , max 8 , IQR $5 ; n=11$ ); government (median 4.5, min 0 , $\max 11$, IQR $6.25 ; n=14$ ); professional (median 4 , min 0 , $\max 11$, IQR $5 ; n=56$ ); and others (median 3.5, min 1, max 11 , IQR $3.75 ; n=12$ ). When the number of symptoms in not-for-profit websites was compared with the value of the rest of the websites in the SERP (median 4, min 0, max 11, IQR $4 ; n=134)$, the difference was statistically significant ( $P=0.003$ by a two-tailed Mann-Whitney test).

\section{Completeness of information on treatments}

Table 3 lists the treatments that were described by the webpages analysed. Overall, the treatments most frequently mentioned were those related to lifestyle interventions, followed by medications and non-pharmacological treatments (such as cognitive behavioural therapy, physiotherapy, counselling and occupational therapy). There were 70 treatments related to complementary and alternative medicine in total,
A

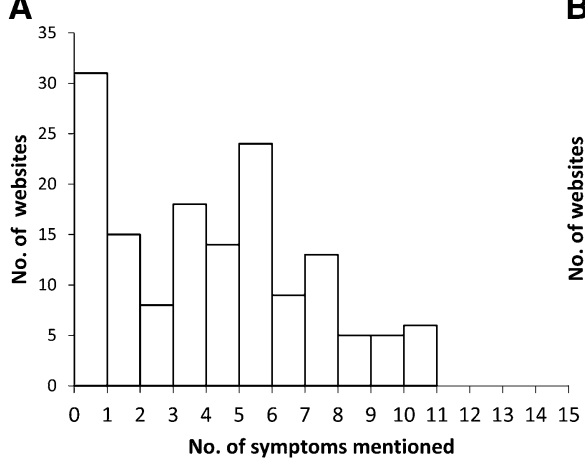

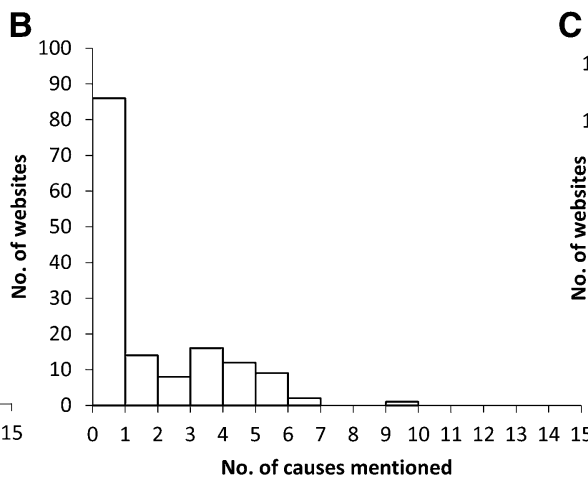

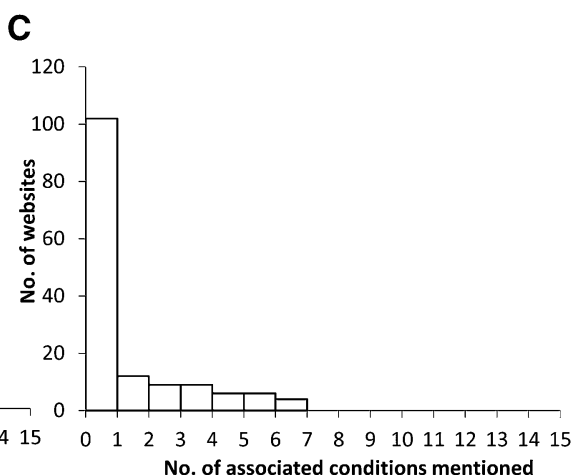

Fig. 2 Frequency distribution of completeness of cover for symptoms (a), causes (b) and associated conditions (c) 
Table 3 Treatment mentioned by the websites

\begin{tabular}{lcllll}
\hline Medications & No. $(\%)$ & Non-pharmacological & No. $(\%)$ & Lifestyle & No. (\%) \\
\hline Anti-depressants & $57(39 \%)$ & Cognitive behavioural therapy & $40(27 \%)$ & Exercise & $77(52 \%)$ \\
Painkillers & $53(36 \%)$ & Physiotherapy & $37(25 \%)$ & Relaxation/stress management & $53(36 \%)$ \\
Anti-convulsants & $42(28 \%)$ & Counselling & $19(13 \%)$ & Sleep hygiene & $38(26 \%)$ \\
Muscle relaxants & $25(17 \%)$ & Occupational therapy & $13(9 \%)$ & Diet & $33(22 \%)$ \\
Anti-inflammatory & $16(11 \%)$ & & & Pacing & $26(18 \%)$ \\
Sleeping pills & $15(10 \%)$ & Complementary/alternative & No. (\%) & Education & 22(15\%) \\
trigger point injection & $11(7 \%)$ & Various & $57(39 \%)$ & Heat & $19(13 \%)$ \\
Anti-psychotic & $7(5 \%)$ & Supplements & $13(9 \%)$ & Mindfulness/meditation & \\
& & & & Cold & $6(4 \%)$ \\
\hline
\end{tabular}

Data indicate the number of websites mentioning the specific item $(n=148)$. Only items that were described at least five times were included in the analysis. Percentages (in parentheses) are calculated on the total number of websites (148)

including acupuncture, massage, relaxation, chiropractic, balneotherapy and others. Thirteen websites described various supplements.

\section{Analysis of standard health information quality measures}

The JAMA score of the 148 websites ranged between 0 and 4 , with a median of 2 , IQR, 2 . Only 63 webpages $(43 \%)$ met the quality threshold of $\geq 3$ [16]. Seventy-nine websites (53\%) displayed the HONcode stamp, whilst only 5/148 (3\%) had the UK NHS IS stamp. Mean readability was grade 9 (median $9, \min 1, \max 18$, IQR 3 ), with only $8 \%$ websites meeting the recommended readability grade 6 . There were no differences in readability between websites from different typologies, with the exception of news (median 6, min 6, max 11, IQR 1) which had a significantly better readability ( $P=0.004$ by two-tailed Kruskal-Wallis multiple comparison test) compared to professional websites (median 10, min 3, max 18, IQR 2). News also had higher readability when compared with the rest of the search $(P<0.001$ by two-tailed Mann-Whitney's test).

\section{Ranking by Google}

As users often only look at the first webpages listed in the SERP, we analysed the completeness of information in terms of Google ranking. Figure 3 shows the completeness,
Fig. 3 Completeness of information in the top ten webpages ranked by Google. Data are median, IQR, minimum and maximum. The $P$ value indicates the level of significance when comparing websites ranking $1-10(n=9)$ to the rest (11-200; $n=139)$ using a twotailed Mann-Whitney test. NS, not significant $(P>0.05)$
A

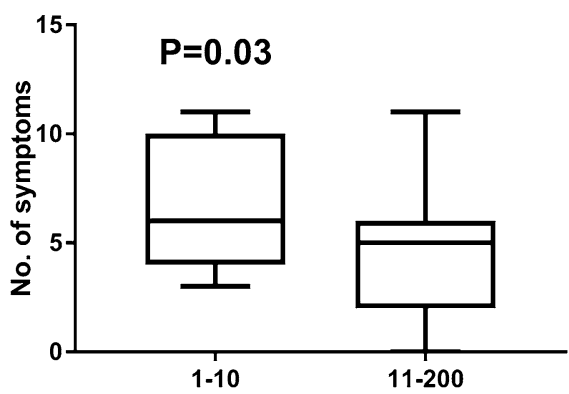

C

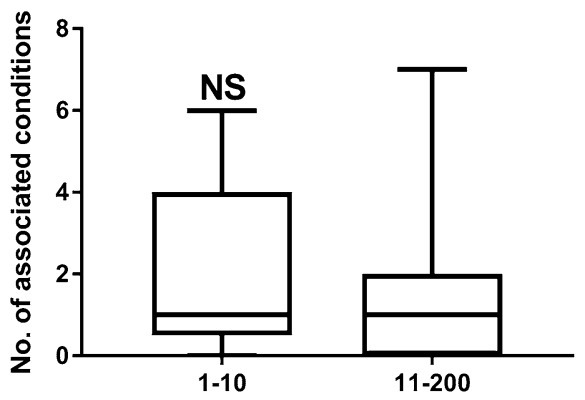

B

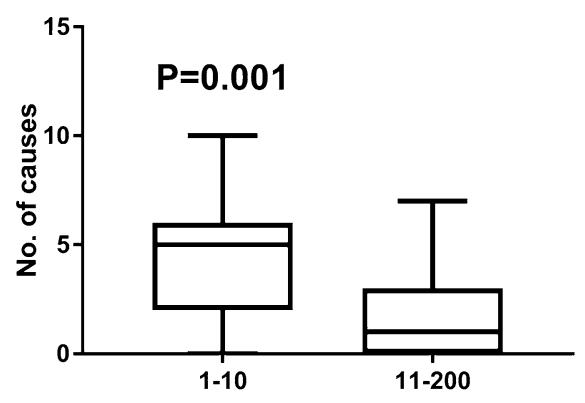

D

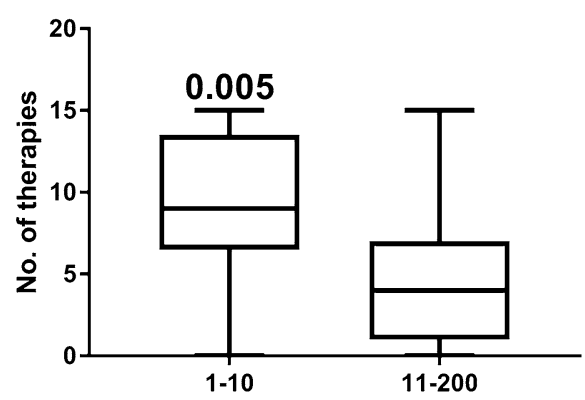


in relation to symptoms (A), causes (B), associated conditions (C) and therapies (D) listed by the top ten websites in comparison to those in the rest of the SERP (11-200). It is clear that, in general, Google ranks webpages providing more complete information highly. On the other hand, there was no difference between the top ten websites compared to the rest of the SERP in terms of JAMA score or in the proportion of websites mentioning complementary and alternative medicine as a therapy (not shown). Readability was slightly higher in the top ten results compared to the rest of the search: top 10 , (median $8, \min 4, \max 10, \mathrm{IQR} 2.5$ ) versus $11-200$ (median $8, \min 1, \max 18$, IQR 3); $P=0.044$ by a two-tailed Mann-Whitney test.

\section{Discussion}

This study investigated the quality of information available online in relation to fibromyalgia, primarily assessing the completeness of content. We found that the completeness of content (symptoms, causes, associated conditions and treatment management) was poor both within and across webpages, consistent with the findings of a previous study [1]. Our findings suggest that fibromyalgia webpages may misinform those that rely on them and we make suggestions of ways online information can be improved.

As the mechanism of fibromyalgia is not fully understood, one might expect that webpages would report that the cause of fibromyalgia is still unknown and that neurological and endocrinological conditions, which are thought to have a central role [7, 22], would be frequently mentioned. However, this was not the case. Many of the ten possible causes listed in webpages were consistent with existing literature [7], but top findings did not mention 'idiopathic' as a cause, hormonal causes accounted for only $7 \%$ of causes mentioned and neurological causes were not listed. Endocrinological and neurological disorders accounted for just $13 \%$ of all associated conditions listed. This lack of clarity may result in patients attributing their illness to something unrelated and potentially withdrawing from activities they consider may be causing or exacerbating their illness.

We found a total of 12 symptoms reported across webpages and have the most complete coverage. Fibromyalgia is reported to be associated with as many as 41 symptoms [23]. However, we found that $21 \%$ of websites mentioned only zero to one symptoms. The lack of symptom reporting may thus lead those with fibromyalgia to unnecessarily return to their physician, requesting further assessments, and for some to suspect they have fibromyalgia when in fact they do not. Due to the complexity of symptoms associated with fibromyalgia, and their crossover with other conditions (for example, multiple sclerosis or lupus), webpages may also wish to report what alternative conditions need to be excluded prior to a diagnosis of fibromyalgia.

The variation in the number and frequency of reported symptoms found within our study may reflect changes to the diagnostic criteria for fibromyalgia [23, 24]. Whist there is no gold-standard assessment criteria, physicians are currently advised to consider a diagnosis of fibromyalgia when widespread pain persists for 3 months or more and symptom manifestation demonstrates challenges in six areas: fatigue, waking unrefreshed, cognitive symptoms, headaches, abdominal pain and depression [23, 24]. We found that over half of the symptoms mentioned in webpages were pain, fatigue, 'fibro fog' and muscle stiffness. These symptoms have been well reported in the literature $[7,13]$ and, with the exclusion of muscle stiffness, all are present in current diagnostic considerations [24]. However, some symptoms associated with fibromyalgia (for example, [23, 24]), were rarely, if at all, discussed in online health information including: muscle weakness, chest pain, fever, wheezing, Raynaud's phenomenon, hives/welts, heartburn, oral ulcers, seizures, shortness of breath, loss of appetite, rash, easy bruising and hair loss.

There is currently no cure for fibromyalgia and its treatment focuses on increasing patient functioning and alleviating pain and other symptoms [25]. As such, patient engagement in the management of fibromyalgia may be primarily improved with greater awareness of symptom manifestation. A lack of reporting of the full range of potential symptoms may have further implications for patients. Some argue that clinical and employment outcomes can be improved with greater public awareness and education [11], the Internet being one mode of public education [26]. Patients already experience high levels of stigma from employers [27] and the general public $[10,12]$. Whilst the Internet could play a role in reducing this stigma and educating the wider public about the condition, the currently limited information available may reduce access to accurate information, perpetuating stigma and leading to frustration for patients.

A second aspect is the information quality of websites according to standard indicators. The majority of studies of health information on fibromyalgia were included in research on chronic pain and found that quality and readability were poor and lacked information surrounding the completeness of fibromyalgia [17, 28]. However, potential indicators of quality were not suggested. We did not find HONcode stamps or JAMA scores to be reliable predictors of content quality or accessibility. In terms of readability, only $8 \%$ of websites meet the recommended reading grade of 6 [28], compared to $16 \%$ in a 2011 study [1]. This indicates that this aspect of information quality regulation has not improved.

Finally, we found the most complete webpages tend to be those from not-for-profit organisations. Patient education is 
recommended across treatment guidelines produced by the American Pain Society, the Canadian Pain Society and the Association of the Scientific Medical Societies in General European League Against Rheumatism [29]. The revised EULAR recommend patient education in the primary instance, with additional treatments considered based on patient needs [5]. As we found not-for-profit webpages to be the most complete, these may be pointed out to future patient as a useful resource. Our findings also suggest the Google algorithm may rank webpages according to the completeness of information. Therefore, improving completeness of content is not only important for fibromyalgia patients, but also for developers seeking to improve the ranking of their websites. The results of this research, in line with pre-existing recommendations $[16,26,28]$, suggesting that future quality assessments may wish to include detailed reviews of content completeness.

Some limitations must be acknowledged with regard to this study. Firstly, only one key word, 'fibromyalgia', was searched. Future research may wish to expand the number of key terms and search engines/platforms used. Engagement with patients may aid in this. Secondly, due to the nebulous nature of fibromyalgia [10], this study was limited as we did not assess scientific accuracy, instead retaining a focus on the total number of symptoms, causes, associated conditions and treatment reported within and across webpages. As the understanding of fibromyalgia grows, more structured research may be completed. In particular, the classification used for recording treatments in this study does not make any judgement of the level of evidence (in terms of evidence-based medicine) for the efficacy of each treatment. For instance, we have put both acupuncture and balneotherapy under complementary and alternative medicine, although the level of evidence for them may be different. Although there are reports that some of them may be useful, both are given a "weak" recommendation by the European League Against Rheumatism (EULAR) guidelines [5], and the current UK National Institute for Clinical Excellence (NICE) described acupuncture as complementary or alternative treatment ("some people find that certain treatments help them to relax and feel less stressed, allowing them to cope with their condition") and hydro/balneotherapy can be used to help cope with the pain of fibromyalgia [30].

In conclusion, our study shows that the completeness of online information with respect to symptoms, causes, associated conditions and treatment of fibromyalgia is poor and content may be inaccessible and open to misinterpretation. The study suggests potential indicators of website quality and completeness, and provides recommendations for organisations whose websites have pages aimed at informing patients with fibromyalgia. Improvements to these areas may begin with improved reporting of symptoms associated with the condition, and this information may aid patients in contributing to treatment decisions. Improving content with respect to symptoms may lessen the stigma these individuals are known to face by helping the general public understand associated limitations. Webpage developers may improve this information by potentially drawing from listed symptoms associated with early fibromyalgia diagnostic criteria. They may wish to engage with patients in the write-up of webpages to ensure that as little technical jargon as possible is used and content is accessible. The information quality indicators identified here have the potential to be used in connecting patients and the general public with quality information, leading to improvements surrounding clinical outcomes, patient engagement, harm reduction and public support.

Author contributions DB, JE and PG designed the study; DB, MF and PG analysed the data; DB, MF, JE and PG wrote the manuscript. All authors read and approved the final version of the manuscript.

Funding The authors have no funding to declare.

\section{Compliance with ethical standards}

Conflict of interest Deepika Basavakumar declares that he/she has no conflict of interest. Mirika Flegg declares that he/she has no conflict of interest. Jessica Eccles declares that he/she has no conflict of interest. Pietro Ghezzi declares that he/she has no conflict of interest.

Ethical approval This article does not contain any studies with human participants or animals performed by any of the authors.

Open Access This article is distributed under the terms of the Creative Commons Attribution 4.0 International License (http://creativeco mmons.org/licenses/by/4.0/), which permits unrestricted use, distribution, and reproduction in any medium, provided you give appropriate credit to the original author(s) and the source, provide a link to the Creative Commons license, and indicate if changes were made.

\section{References}

1. Daraz L, Macdermid JC, Wilkins S, Gibson J, Shaw L (2011) The quality of websites addressing fibromyalgia: an assessment of quality and readability using standardised tools. BMJ Open 1(1):e000152. https://doi.org/10.1136/bmjopen-2011-000152

2. Boulton $\mathrm{T}$ (2018) Nothing and everything: fibromyalgia as a diagnosis of exclusion and inclusion. Qual Health Res:1049732318804509. https://doi.org/10.1177/1049732318 804509

3. Hauser W, Fitzcharles MA (2018) Facts and myths pertaining to fibromyalgia. Dialogues Clin Neurosci 20(1):53-62

4. Lichtenstein A, Tiosano S, Amital H (2018) The complexities of fibromyalgia and its comorbidities. Curr Opin Rheumatol 30(1):94-100. https://doi.org/10.1097/BOR.0000000000000464

5. Macfarlane GJ, Kronisch C, Dean LE, Atzeni F, Hauser W, Fluss E, Choy E, Kosek E, Amris K, Branco J, Dincer F, Leino-Arjas P, Longley K, McCarthy GM, Makri S, Perrot S, Sarzi-Puttini P, 
Taylor A, Jones GT (2017) EULAR revised recommendations for the management of fibromyalgia. Ann Rheum Dis 76(2):318-328. https://doi.org/10.1136/annrheumdis-2016-209724

6. Talotta R, Bazzichi L, Di Franco M, Casale R, Batticciotto A, Gerardi MC, Sarzi-Puttini P (2017) One year in review 2017: fibromyalgia. Clin Exp Rheumatol 35(Suppl 105 (3):6-12

7. Borchers AT, Gershwin ME (2015) Fibromyalgia: a critical and comprehensive review. Clin Rev Allergy Immunol 49(2):100-151. https://doi.org/10.1007/s12016-015-8509-4

8. National Health Service UK (2018) Fibromyalgia-NHS. https:// www.nhs.uk/conditions/fibromyalgia/. Archived at: https://web. archive.org/web/20180903095536/https://www.nhs.uk/conditions /fibromyalgia/. Accessed 19 Feb 2019

9. Patient.info (2018) Fibromyalgia (Fibrositis). Fibromyalgia symptoms and treatment. @ patient. https://patient.info/doctor/fibro myalgia-pro. Archived at: https://web.archive.org/web/20180 903095812/https://patient.info/doctor/fibromyalgia-pro

10. Lempp HK, Hatch SL, Carville SF, Choy EH (2009) Patients' experiences of living with and receiving treatment for fibromyalgia syndrome: a qualitative study. BMC Musculoskelet Disord 10:124. https://doi.org/10.1186/1471-2474-10-124

11. Blom D, Thomaes S, Kool MB, van Middendorp H, Lumley MA, Bijlsma JW, Geenen R (2011) A combination of illness invalidation from the work environment and helplessness is associated with embitterment in patients with FM. Rheumatology 51(2):347-353

12. Kool MB, van Middendorp H, Boeije HR, Geenen R (2009) Understanding the lack of understanding: invalidation from the perspective of the patient with fibromyalgia. Arthritis Rheum 61(12):1650-1656. https://doi.org/10.1002/art.24922

13. Jones GT, Atzeni F, Beasley M, Fluss E, Sarzi-Puttini P, Macfarlane GJ (2015) The prevalence of fibromyalgia in the general population: a comparison of the American College of Rheumatology 1990, 2010, and modified 2010 classification criteria. Arthritis Rheumatol 67(2):568-575. https://doi.org/10.1002/art.38905

14. The UK's most searched illnesses (2015) TheWeek. https://www. theweek.co.uk/68082/the-uks-most-searched-illnesses, Archived at: https://web.archive.org/web/20190219122254/https://www. theweek.co.uk/68082/the-uks-most-searched-illnesses. Accessed 19 feb 2019

15. Chapman L, Brooks C, Lawson J, Russell C, Adams J (2017) Accessibility of online self-management support websites for people with osteoarthritis: a text content analysis. Chronic Illn:1742395317746471. https://doi.org/10.1177/1742395317 746471

16. Silberg WM, Lundberg GD, Musacchio RA (1997) Assessing, controlling, and assuring the quality of medical information on the Internet: Caveant lector et viewor-let the reader and viewer beware. JAMA 277(15):1244-1245

17. Washington TA, Fanciullo GJ, Sorensen JA, Baird JC (2008) Quality of chronic pain websites. Pain Med 9(8):994-1000. https ://doi.org/10.1111/j.1526-4637.2008.00419.x

18. Boyer C, Selby M, Scherrer JR, Appel RD (1998) The health on the net code of conduct for medical and health websites. Comput Biol Med 28(5):603-610
19. Statista (2018) https://www.statista.com/statistics/280269/marke t-share-held-by-search-engines-in-the-united-kingdom/. Archived at: https://web.archive.org/web/20180907131821/https://www. statista.com/statistics/280269/market-share-held-by-search-engin es-in-the-united-kingdom/. Accessed 7 Sep 2018

20. Arif N, Ghezzi P (2018) Quality of online information on breast cancer treatment options. Breast 37:6-12. https://doi. org/10.1016/j.breast.2017.10.004

21. Manley L, Ghezzi P (2018) The quality of online health information on breast augmentation. J Plast Reconstr Aesthet Surg. https ://doi.org/10.1016/j.bjps.2018.07.023

22. Woolf CJ (2011) Central sensitization: implications for the diagnosis and treatment of pain. Pain 152(3 Suppl):S2-S15. https:// doi.org/10.1016/j.pain.2010.09.030

23. Wolfe F, Clauw DJ, Fitzcharles MA, Goldenberg DL, Katz RS, Mease P, Russell AS, Russell IJ, Winfield JB, Yunus MB (2010) The American College of Rheumatology preliminary diagnostic criteria for fibromyalgia and measurement of symptom severity. Arthritis Care Res (Hoboken) 62(5):600-610. https://doi. org/10.1002/acr.20140

24. Wolfe F, Clauw DJ, Fitzcharles MA, Goldenberg DL, Hauser W, Katz RL, Mease PJ, Russell AS, Russell IJ, Walitt B (2016) 2016 Revisions to the 2010/2011 fibromyalgia diagnostic criteria. Semin Arthritis Rheum 46(3):319-329. https://doi.org/10.1016/j. semarthrit.2016.08.012

25. Hoffman DL, Dukes EM (2008) The health status burden of people with fibromyalgia: a review of studies that assessed health status with the SF-36 or the SF-12. Int J Clin Pract 62(1):115-126. https://doi.org/10.1111/j.1742-1241.2007.01638.x

26. Dutta-Bergman MJ (2004) The impact of completeness and web use motivation on the credibility of e-health information. J Commun 54(2):253-269

27. Blom D, Thomaes S, Kool MB, van Middendorp H, Lumley MA, Bijlsma JW, Geenen R (2012) A combination of illness invalidation from the work environment and helplessness is associated with embitterment in patients with FM. Rheumatology 51(2):347353. https://doi.org/10.1093/rheumatology/ker342

28. Kaicker J, Debono VB, Dang W, Buckley N, Thabane L (2010) Assessment of the quality and variability of health information on chronic pain websites using the DISCERN instrument. BMC Med 8:59. https://doi.org/10.1186/1741-7015-8-59

29. Thieme K, Mathys M, Turk DC (2017) Evidenced-based guidelines on the treatment of fibromyalgia patients: Are they consistent and if not, why not? Have effective psychological treatments been overlooked? J Pain 18(7):747-756

30. NICE. https://www.nhs.uk/conditions/fibromyalgia/treatment/. Archived at: https://web.archive.org/web/20190214131910/https ://www.nhs.uk/conditions/fibromyalgia/treatment/. Accessed 19 Feb 2019

Publisher's Note Springer Nature remains neutral with regard to jurisdictional claims in published maps and institutional affiliations. 